\title{
Prospectiva hacia el Aprendizaje Móvil en Estudiantes Universitarios
}

\author{
Agustín Lagunes-Domínguez, Carlos A. Torres-Gastelú, Joel Angulo-Armenta y \\ Miguel Á. Martínez-Olea \\ Universidad Veracruzana, Facultad de Contaduría y Administración, Camino a Dos Ríos Km. 1, \\ Ixtaczoquitlán, México. (e-mail: aglagunes@uv.mx, ctorres@uv.mx, joel.angulo@itson.edu.mx; \\ zs10002798@estudiantes.uv.mx)
}

Recibido Jun. 17, 2016; Aceptado Ago. 29, 2016; Versión final Oct. 3, 2016, Publicado Feb. 2017

\section{Resumen}

Se ha realizado un estudio para determinar si los estudiantes de una universidad colombiana y una mexicana tienen el equipo, la disponibilidad de tiempo y la disposición para el aprendizaje móvil dentro de sus clases. Para ello se diseñó y validó un instrumento que se aplicó a los estudiantes del área de Administración y de Sistemas en una universidad pública colombiana y otra universidad pública mexicana. Los resultados muestran que los estudiantes colombianos y mexicanos cuentan con dispositivos móviles y están dispuestos a utilizarlos para aprendizaje sus clases. Asimismo, se encontró que no hay diferencia entre los estudiantes del área de Administración y los de Sistemas, ambos aceptan el aprendizaje móvil por igual. Desde la perspectiva de los estudiantes, existe la factibilidad técnica, de tiempo y de disposición para el aprendizaje móvil en ambas universidades.

Palabras clave: aprendizaje móvil; internet en clases; enseñanza-aprendizaje; enseñanza universitaria

\section{Exploration toward Mobile Learning in University Students}

\begin{abstract}
A study has been done to determine the disposition of university students of Colombia and Mexico to mobile learning, analyzing the type of mobile equipment they have, and whether they have or not the willingness and the required time to use this technology. To achieve this, an instrument was designed and evaluated with a group of students of administration and computer sciences of a Colombian university and a Mexican university. The results show that Colombian and Mexican students have similar mobile devices and they are willing to use them as a means of learning. Besides, it was found that both the administration students and the computer science students equally accept the use their mobile devices for learning. From the students' perspective, they have the technical means, the time and the willingness for mobile learning in both universities.
\end{abstract}

Keywords: mobile learning; Internet in the classroom; teaching - learning process; university education 


\section{INTRODUCCIÓN}

La integración de las Tecnologías de la Información y Comunicación (TIC) en la sociedad, ha venido siendo una de las líneas de investigación más recurrentes en las últimas dos décadas, por citar algunos aportes del estado que guardan, sobresalen (Cho, 2015), su disponibilidad, acceso y su uso (Abbott y Azerikatoa, 2014), su integración y las estrategias para su implementación (Ani et al., 2016) y cómo medir el impacto que estas han tenido en la sociedad (Bandyopadhyay, 2015). Sobre la base de las consideraciones anteriores, en el sector educativo la incorporación de las nuevas tecnologías en los procesos de enseñanza y aprendizaje ha crecido de manera exponencial, significa entonces que la educación superior ha sufrido cambios significativos a través de experiencias documentadas de las TIC en educación superior como la incorporación de multimedia y juegos, el aprendizaje en línea, los Entornos Virtuales de Aprendizaje (EVA), los Recursos Educativos Abiertos (REA) (Hernández et al., 2014; Lechuga et al., 2014; Mejía y López, 2016).

Precisando de una vez, aunque existe una amplia literatura en cuanto a la utilización de las TIC en educación superior, como son propuestas de modelos tecno pedagógicos, nuevas metodologías didácticas, entornos no convencionales de aprendizaje, estudios comparativos entre países e instituciones de educación superior, entre otros (Lagunes-Dominguez et al., 2015), aún hay temas que están pendientes de estudiar y documentar a profundidad para demostrar su factibilidad y aceptación, entre estos temas se encuentra el aprendizaje móvil. El aprendizaje móvil, también llamado 'm-learning' ofrece métodos modernos de apoyo al proceso de aprendizaje mediante el uso de dispositivos móviles, tales como las computadoras portátiles, las tabletas, los reproductores MP3 y los teléfonos inteligentes (UNESCO, 2016).

En los últimos años el aprendizaje móvil se está convirtiendo en una de las soluciones a los problemas que enfrenta el sector educativo (Huang et al., 2016; Su y Cheng, 2015). Por ello, el programa de la UNESCO se basa en un número cada vez mayor de iniciativas orientadas a estudiar de qué manera las tecnologías móviles pueden ayudar a la consecución de la meta "Educación para Todos" (UNESCO, 2016). El aprendizaje móvil es la oportunidad de aprovechar las bondades de los dispositivos móviles para el proceso de enseñanza - aprendizaje y adquirió auge cuando la UNESCO inició los trabajos para orientar, normal y fortalecer el aprendizaje móvil alrededor del mundo.

En el año de 2012, la UNESCO generó una serie de documentos sobre aprendizaje móvil, el propósito era lograr una mayor comprensión de cómo las tecnologías móviles pueden ser utilizadas para mejorar el acceso, la equidad y la calidad de la educación en todo el mundo (Jara et al., 2012). En ese mismo sentido, la UNESCO dividió los estudios en cinco regiones: África y el Medio Oriente, América Latina, América del Norte, Asia, y Europa. Entre los documentos enfocados a la región de América Latina se encuentran "Aprendizaje móvil para docentes en América Latina" (Jara et al., 2012), "Activando el aprendizaje móvil en América Latina" (Lugo y Schurmann, 2012) y "Aprendizaje móvil para docentes" (Lugo y Schurmann, 2012).

El primer documento presenta un análisis de 21 iniciativas de aprendizaje móvil en América Latina, la cuales se distribuyen con 5 en Chile, 4 en Argentina y la misma cantidad en México, 3 en Colombia, y con una iniciativa El Salvador, Honduras, Paraguay y Perú, existiendo un proyecto llamado Kantoo orientado a lecciones en inglés para teléfonos móviles que se aplica en diversos países (Jara et al., 2012). De las 21 iniciativas que presenta la UNESCO, solo seis de ellas están dirigidas a la educación superior y las tres que considera las más importantes "Puentes Educativos" en Chile, "Raíces de Aprendizaje Móvil" en Colombia, y "Entorno Móvil Interactivo de Aprendizaje" en Argentina, ninguna de ellas está dirigida a universidades, por lo cual aún hay mucho camino por recorrer en este nivel educativo (Jara et al., 2012).

En el año 2013, la UNESCO generó tres documentos muy importantes 1) "Aprendizaje móvil y políticas" (Vosloo, 2013), 2) "Directrices para las políticas de aprendizaje móvil" (UNESCO, 2013) y 3) "El futuro del aprendizaje móvil, implicaciones para la planificación y la formulación de políticas" (Shuler et al., 2013). En esta serie de documentos se hicieron aportaciones significativas al establecimiento de políticas educativas y se basó en siete principios rectores: aprovechar las inversiones existentes, localizar las políticas, apoyar las normas técnicas abiertas, promover la cooperación intersectorial y las alianzas de múltiples interesados, establecer políticas a todos los niveles, revisar y actualizar las políticas existentes y el último, garantizar una educación incluyente. Como resultado de lo descrito, la UNESCO (2015) publica el libro "La lectura en la era móvil" (West y Ei Chew, 2015), sobre cómo llevar la lectura a los países que no están desarrollados, mediante la propuesta de los libros digitales y el teléfono móvil como herramienta. Después del análisis de la serie de documentos generados por la UNESCO entre el 2012 y el 2015, se hace necesaria una revisión de la literatura en aprendizaje móvil. Al igual que las investigaciones sobre las TIC, el trabajo realizado en aprendizaje móvil es muy variado, en diferentes contextos y desde luego en diferentes países alrededor del mundo. 
El análisis que se presenta sobre la literatura en aprendizaje móvil ha sido dividido en 1) La implementación y el impacto del aprendizaje móvil y sus efectos, 2) Cómo se ha unido el aprendizaje móvil con la investigación, 3) La percepción que tienen los profesores y estudiantes sobre esta propuesta, y 4) La factibilidad de su implementación en las instituciones de educación superior. En cuanto a la implementación, una de las lecturas obligadas es la de la American Library Association, donde explica la tendencias, la accesibilidad, ecosistemas y la creación de contenidos, en este libro se expone cómo se está haciendo que el aprendizaje sea más fácil para todas las edades, así mismo indica que la unión de servicios y dispositivos crea un nuevo ecosistema de aprendizaje y concluye aterrizando la oportunidad que tienen las bibliotecas para implantar el aprendizaje móvil (Hennig, 2016).

Por su parte, Montrieux et al., (2015) realizaron un estudio cuantitativo exploratorio acerca de la introducción de los dispositivos móviles en el aula, para ello, se implantó el aprendizaje móvil y posteriormente se registró la opinión de los estudiantes y profesores. Este estudio arrojó tres aportes principales. 1) El primero es referente a cómo se concibe al profesor en el proceso de enseñanza; para ello, los investigadores agruparon las opiniones en profesores instrumentales (67\%) y profesores innovadores (33\%); 2) El segundo aporte es acerca de las prácticas de aprendizaje, tanto los profesores como los estudiantes consideran el valor añadido de los dispositivos en referencia a la facilidad de uso, la velocidad de acceso de diferentes materiales de aprendizaje; la capacidad de ser capaz de buscar al instante información adicional, de tomar fotografías e integrar las notas, y hasta la reducción en el de peso de sus mochilas; y 3) El tercer, es respecto a las condiciones que apoyan estas prácticas de enseñanza y aprendizaje, los investigadores las clasificaron en dos, las condiciones materiales y la necesidad de profesionalización. En la primera consideran que están abandonados por las editoriales, ya que los materiales son creados para un ambiente normal y transcritos a los dispositivos, esto es, no aprovechan las bondades del dispositivo para un aprendizaje diversificado. Así mismo, los profesores no han asumido el papel innovador que amerita este aprendizaje, por tal motivo, los autores concluyen que en materiales y capacitación hay mucho por hacer.

Otros trabajos de implementación del aprendizaje móvil son casos de estudio de la integración de teléfonos móviles, entre ellos se encuentra el desarrollado en Sri Lanka en la India sobre la formación de profesores en ciencias (Ekanayake y Wishart, 2015). En este mismo sentido, hay autores que proponen que se estudie la motivación de los estudiantes para su implementación (Kilis, 2013) y en este tenor, hay estudios sobre la motivación que tienen los adultos por esta alternativa como el trabajo de Faizal-Hashim et al. (2015), el cual cabe mencionar demostró que los estudiantes adultos tienen una fuerte preferencia para adoptar el aprendizaje móvil con fines de aprendizaje. Lo anterior apoya a la factibilidad de su implementación en educación superior.

En cuanto al uso del aprendizaje móvil en investigación, hay diversos autores que han estado documentando el tema (Al-Zahrani y Laxman, 2015; Baran, 2014; Cheng et al., 2016). Los autores estudian cómo aplicar esta tecnología para trabajos de investigación, aunque cabe destacar que algunos de ellos (Al-Zahrani y Laxman, 2015; Baran, 2014) cambian el enfoque y realizan una revisión literaria de artículos de investigación sobre el aprendizaje móvil dando una visión más amplia de los investigado en este tema. Algunas evidencias empíricas que se aproximan mucho al presente estudio es el de LópezHernández y Silva-Pérez (2016), quienes analizaron la aceptación de los dispositivos móviles para el aprendizaje en educación superior en España. La importancia de este estudio radica en que los estudiantes pueden tomar los dispositivos móviles en cualquier lugar que quieran ejecutar tareas o continuar su aprendizaje más allá del aula en concordancia con Yu-Chang e Yu-Hui (2015).

De acuerdo a los razonamientos que se han venido realizando, hay aportaciones muy interesantes (DeArriba-Pérez et al., 2015), que van más allá de determinar percepciones de estudiantes, de la aplicación e implementación del aprendizaje móvil; los autores argumentan algunos algoritmos de recolección y tratamiento de información, que permitan obtener perfiles de los estudiantes y ofrecer, a partir de los resultados, un servicio personalizado a todos a ellos mismos, lo cual es una aportación muy importante porque se piensa en su heterogeneidad. Por lo anterior, esta investigación es necesaria para mostrar la visión que tienen los estudiantes de Colombia y México acerca de la utilización de los dispositivos móviles en su aprendizaje y la cual servirá como base para un estudio similar al de DeArribaPérez, Caeiro-Rodríguez y Santos-Gago (2015) en estos países.

\section{MATERIALES Y MÉTODOS}

Para la investigación se eligieron dos universidades públicas, una de Colombia y otra de México. Estas universidades fueron elegidas a través de un muestreo no probabilístico y con el criterio por conveniencia debido a que, ambas universidades deseaban iniciar con proyectos educativos usando dispositivos móviles y además sus características son similares y también se eligieron las áreas de Administración y Sistemas porque eran las únicas afines en ambas universidades. El objetivo del estudio fue determinar si 
los estudiantes de una universidad colombiana y una mexicana tienen el equipo, la disponibilidad de tiempo y la disposición para el aprendizaje móvil dentro de sus clases, y contrastar los resultados entre ambas universidades. La investigación fue cuantitativa de corte descriptivo aplicando una encuesta, para ello se diseñó un instrumento de siete preguntas que fue valorado por 10 expertos, se recibieron los cambios sugeridos, se realizaron las correcciones y una vez liberado el instrumento fue aplicado. La aplicación de la prueba de Alfa Cronbach fue de 90\%, indicando con ello un valor aceptable de confiabilidad.

En el caso de la Universidad Colombiana la población se distribuyó en 624 estudiantes de Administración y 351 de Sistemas, dando un total de 975 participantes. Los participantes de la Universidad Mexicana fueron 641 estudiantes, distribuidos en 422 de Administración y 219 de Sistemas. Se utilizó la fórmula del muestreo aleatorio simple para poblaciones finitas (Munch-Galindo y Angeles, 1998), dicha fórmula permitió determinar el tamaño de la muestra y quedó representada de la siguiente manera:

$$
\eta_{\theta}=\frac{\sigma^{2} Z_{(\infty / 2)}^{2}}{e^{2}}
$$

Una vez aplicada la fórmula para la Universidad Colombiana la muestra fue de 179 estudiantes de Administración y 144 estudiantes de Sistemas, mientras que para la Universidad Mexicana fue de 156 estudiantes de Administración y 116 estudiantes de Sistemas. La elección de los participantes fue mediante un muestreo aleatorio estratificado por periodo que cursaban los estudiantes.

La edad de los estudiantes de la Universidad Colombiana estuvo en un rango de 17 y 32 años, mientras que en la Universidad Mexicana era entre 17 y 31 años. En general en ambas universidades, poco más del $65 \%$ de los estudiantes eran mujeres y el resto varones. En cuanto a la validación de la captura esta se logró mediante tres capturas por separado y posteriormente la unión de las mismas en una hoja de cálculo aplicando la fórmula:

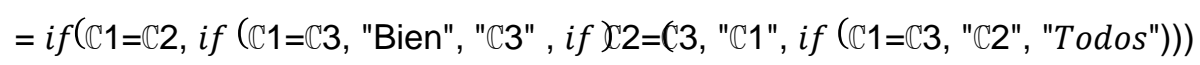

La fórmula indica cuál de las capturas es diferente, "Todos" si las tres están diferentes; "C1", "C2" o "C3" si alguna de ellas está diferente; el tercer caso es "Bien" cuando todas coinciden. En el primer y segundo caso, se recurre al instrumento original para corregir y que todos los reactivos de la captura indiquen "Bien". Una vez determinada la validez de la captura, se importaron los datos en SPSS.

\section{RESULTADOS Y DISCUSIÓN}

Para analizar los resultados se presentan cinco tablas, con ellas se busca conocer quienes tienen dispositivos móviles y de qué tipo son; también se desea saber quiénes viajan en autobús a la universidad y el tiempo del trayecto; finalmente, la disponibilidad que tienen los estudiantes para usar los dispositivos móviles para sus clases.

Tabla 1: Estudiantes que poseen dispositivo móvil.

\begin{tabular}{|l|c|c|}
\hline \multirow{2}{*}{} & Universidad Colombiana & Universidad Mexicana \\
\cline { 2 - 3 } & Porcentaje & Porcentaje \\
\hline SI & 98.45 & 98.16 \\
\hline NO & 1.55 & 1.84 \\
\hline Total & 100.00 & 100.00 \\
\hline
\end{tabular}

La Tabla 1 muestra la gran similitud que existe entre ambas universidades en el uso de algún dispositivo móvil. La diferencia estriba en la variedad de dispositivos que utilizan, como se observa en la Tabla 2 los estudiantes utilizan más de un dispositivo móvil, y mientras en la Universidad Colombiana utilizan más el Smartphone, en la Universidad Mexicana utilizan con más frecuencia la computadora portátil. En general en ambas universidades se utilizan más la computadora portátil y el Smartphone.

Con el objetivo de determinar si los estudiantes podrían utilizar los dispositivos móviles para su aprendizaje durante el trayecto a la universidad, se les cuestionó acerca si viajaban en autobús para llegar a la universidad. La Tabla 3 muestra que los estudiantes de la Universidad Mexicana se trasladan a la universidad más en autobús que los estudiantes colombianos, aunque los dos porcentajes son elevados. 
Tabla 2: Variedad de dispositivos que utilizan los estudiantes.

\begin{tabular}{|l|c|c|}
\hline & Universidad Colombiana & Universidad Mexicana \\
\hline & Porcentaje & Porcentaje \\
\hline Teléfono sencillo & 24.38 & 42.27 \\
\hline Computadora portátil & 73.45 & 64.33 \\
\hline Smartphone & 78.70 & 55.88 \\
\hline Tablet & 20.67 & 17.64 \\
\hline Otro & 2.46 & 1.47 \\
\hline
\end{tabular}

Tabla 3: Uso del autobús para el traslado a la universidad.

\begin{tabular}{|l|c|c|}
\hline \multirow{2}{*}{} & Universidad Colombiana & Universidad Mexicana \\
\cline { 2 - 3 } & Porcentaje & Porcentaje \\
\hline SI & 73.15 & 94.11 \\
\hline NO & 26.85 & 5.89 \\
\hline Total & 100.00 & 100.00 \\
\hline
\end{tabular}

Una vez determinado esto, se deseaba conocer el tiempo que permanecen en el autobús para llegar a la universidad. La Tabla 4 muestra la frecuencia de los tiempos, cabe mencionar que en la Universidad Colombiana el $40 \%$ se lleva entre 31 y 60 minutos de traslado, mientras que el $20.20 \%$ invierte más de una hora de su tiempo. Por lo que respecta a la Universidad Mexicana los porcentajes son similares, $40.78 \%$ entre 31 y 60 minutos de traslado y $28.24 \%$ más de una hora. Finalmente se preguntó a los estudiantes si estarían dispuestos a probar el aprendizaje móvil en sus clases obteniendo como resultado lo mostrado en la Tabla 5.

Tabla 4: Tiempo en el autobús para llegar a la universidad.

\begin{tabular}{|l|c|c|}
\hline & Universidad Colombiana & Universidad Mexicana \\
\hline & Porcentaje & Porcentaje \\
\hline De 5 a 10 minutos & 2.40 & 6.67 \\
\hline De 11 a 20 minutos & 20.40 & 3.53 \\
\hline De 21 a 30 minutos & 16.80 & 20.78 \\
\hline De 31 a 40 minutos & 14.40 & 9.80 \\
\hline De 41 a 50 minutos & 12.80 & 30.98 \\
\hline De 51 a 60 minutos & 12.80 & 0 \\
\hline Más de una hora & 20.40 & 28.24 \\
\hline Total & 100.00 & 100.00 \\
\hline
\end{tabular}

Tabla 5: Estudiantes que estarían dispuestos a usar su dispositivo móvil para tus clases

\begin{tabular}{|l|c|c|}
\hline \multirow{2}{*}{} & Universidad Colombiana & Universidad Mexicana \\
\cline { 2 - 3 } & Porcentaje & Porcentaje \\
\hline SI & 97.53 & 92.65 \\
\hline NO & 2.47 & 7.35 \\
\hline Total & 100.00 & 100.00 \\
\hline
\end{tabular}

Como se puede observar los estudiantes de la Universidad Colombiana tienen mayor disposición a utilizar los dispositivos móviles para sus clases, la diferencia entre las universidades no es significativa y si es un porcentaje muy elevado de aceptación.

Cuando se inició con esta investigación se tenían dos hipótesis, la primera era que "Los estudiantes de la Universidad Colombiana tienen mayor disposición a utilizar los dispositivos móviles en sus clases", se determinó la correlación de acuerdo a Lind et al., (2004) donde indican que un valor entre 0 y 0.5 es una correlación positiva débil, 0.5 es moderada y entre 0.5 y 1 es una correlación positiva fuerte, teniendo como resultado 0.11 lo cual significa que es una correlación positiva débil, esto es, no importa de qué universidad sean los estudiantes, de igual manera aceptan utilizar los móviles para sus clases. La segunda 
hipótesis que se tenía es "Los estudiantes del área de Sistemas tienen mayor aceptación hacia el uso de los móviles en clases que los estudiantes del área de Administración", la correlación determinó un 0.08 por lo cual se aprueba la hipótesis alterna donde no hay diferencias entre la carrera que estudien y su disposición a utilizar los dispositivos móviles en sus clases.

Para complementar la parte cuantitativa se realizó un breve estudio cualitativo, donde los estudiantes indicaban porque trabajar con aprendizaje móvil y si no estaban de acuerdo, por qué no hacerlo. Los resultados fueron muy interesantes, sus respuestas de porque usar el aprendizaje móvil tienen que ver con que pasan mucho tiempo con su celular, porque les atrae y les gusta (esparcimiento), porque sería más fácil, sencillo y entretenido. Por el contrario, ellos indicaron dos razones principales, porque los distraen y porque prefieren lo presencial, estas respuestas son muy reveladoras y deben ser motivo de estudio en una investigación posterior.

\section{CONCLUSIONES}

De acuerdo a la información recabada se concluye que los estudiantes cuentan con los dispositivos móviles en caso que se decida implementar el aprendizaje móvil como lo indica la Tabla 1 con porcentajes mayores a $98 \%$ en ambas universidades.

Los estudiantes en su mayoría, $73.15 \%$ de los colombianos y $94.11 \%$ los mexicanos, viajan en autobús para trasladarse a la universidad y de ellos, el $40 \%$ invierten entre 31 minutos y una hora para su traslado, mientras que más del $20 \%$ dedican más de una hora a esta actividad. Por lo anterior se infiere que los estudiantes de ambas universidades tendrían tiempo suficiente para estudiar o realizar actividades en su dispositivo móvil durante el trayecto a la universidad, si así lo desearan.

Se concluye que no hay diferencia entre la disposición de los estudiantes colombianos y mexicanos de estas dos universidades públicas para utilizar sus dispositivos para sus clases, teniendo un porcentaje de aceptación de $97.53 \%$ de los estudiantes colombianos y $92.65 \%$ de los estudiantes mexicanos. Esta tendencia se mantiene sin importar si los estudiantes son del área de Administración o del área de Sistemas, los resultados arrojan que tienen una diferencia que no es estadísticamente significativa.

Finalmente se concluye que existe la factibilidad técnica -los estudiantes tienen los dispositivos-, la factibilidad de tiempo -los estudiantes invierten mucho tiempo al traslado a la universidad-, y la disposición de los estudiantes por usar los dispositivos móviles en sus clases.

Debido a los resultados mostrados se recomienda implementar algoritmos de recolección y tratamiento de información, que permitan obtener perfiles de los estudiantes y con ello diseñar experiencias educativas atendiendo a las necesidades particulares de cada estudiante de acuerdo a lo propuesto con DeArribaPérez, Caeiro-Rodríguez y Santos-Gago (2015).

\section{REFERENCIAS}

Abbott, P., y Azerikatoa, D. ICT and empowerment to participate: a capability approach, Information Development, 30(4), 321-331 (2014)

Al-Zahrani, H., y Laxman, K. A Critical Meta-Analysis of Mobile Learning Research in Higher Education, Journal of Technology Studies, 41(2), 74-89 (2015)

Ani, E. O., Ugwu, C. O., Nwachukwu, A. I., Obianuko, J. C., Ndaji, G. I., y Maduchie, E. E. Strategies for Enhancing the Utilization of Information and Communication Technology (ICT)-Based Library Resources in Research, Library Philosophy \& Practice, 1-24 (2016)

Bandyopadhyay, A. K. Impact of ICT on Job Satisfaction among University Library Professionals of West Bengal: A Comparison Study, SRELS Journal of Information Management, 52(5), 371-376 (2015)

Baran, E. A Review of Research on Mobile Learning in Teacher Education, Educational Technology \& Society, 17(4), 17-32 (2014)

Cheng, P.-H., Yang, Y.-T. C., Chang, S.-H. G., y Kuo, F.-R. R. 5E Mobile Inquiry Learning Approach for Enhancing Learning Motivation and Scientific Inquiry Ability of University Students, IEEE TRANSACTIONS ON EDUCATION, 59(2), 147-153 (2016) 
Cho, C. M. Is ICT a new essential for national economic growth in an information society?, Government Information Quarterly, 32(3), 253-260 (2015)

DeArriba-Pérez, F., Caeiro-Rodríguez, M., y Santos-Gago, J. M. Knowledge extraction from usage data of mobile devices with educational purposes. 10Th Iberian Conference On Information Systems And Technologies, CISTI 2015 (2015)

Ekanayake, S. Y., y Wishart, J. Integrating mobile phones into teaching and learning: A case study of teacher training through professional development workshops, doi:10.1111/bjet.12131, British Journal of Educational Technology, 46(1), 173-189 (2015)

Faizal-Hashim, K., Tan, F. B., y Rashid, A. Adult learners' intention to adopt mobile learning: A motivational perspective, doi:10.1111/bjet.12148, British Journal of Educational Technology, 46(2), 381-390 (2015)

Hennig, N. Mobile Learning Trends: Accessibility, Ecosystems, Content Creation (Vol. 52): American Library Association (2016)

Hernández, M. R., Rodríguez, V. M., Parra, F. J., y Velázquez, P. Las Tecnologías de la Información y la Comunicación (TICS) en la Enseñanza-Aprendizaje de la Química Orgánica a través de Imágenes, Juegos y Videos, doi:10.4067/S0718-50062014000100005, Formación Universitaria, 7(1), 31-40 (2014)

Huang, C. S., Yang, S. J., Chiang, T. H., y Su, A. Y. Effects of Situated Mobile Learning Approach on Learning Motivation and Performance of EFL Students. Educational Technology \& Society, 19(1), 263-276 (2016)

Jara, I., Claro, M., y Martinic, R. Aprendizaje móvil para docentes en América Latina, UNESCO, 1-50 (2012)

Kilis, S. Impacts of Mobile Learning in Motivation, Engagement and Achievement of Learners: Review of Literature, Gaziantep University Journal of Social Sciences, 12(2), 375-383 (2013)

Lagunes-Dominguez, A., Torres-Gastelú, C. A., Flores-García, M. A., y Rodriguez-Figueroa, A. Comparativo del uso de Tecnologías de la Información y Comunicación (TIC) por Profesores de Dos Universidades Públicas de México, doi:10.4067/S0718-50062015000200003, Formación Universitaria, 8(2), 11-18 (2015)

Lechuga, M., Fernández-Arteaga, A., Ríos, F., y Fernández-Serrano, M. Utilización de Entornos Virtuales (2014)

Educativos y Recursos Educativos Abiertos (OpenCourseWare) en cursos de Ingeniería Química de la Universidad de Granada, España, doi:10.4067/S0718-50062014000400002, Formación Universitaria, 7(4), 3-14 (2014)

Lind, D. A., Marchal, W. G., y Mason, R. D. Estadística para administración y economía, 11 edición, Alfaomega (2004)

López-Hernández, F. A., y Silva-Pérez, M. M. Factores que inciden en la aceptación de los dispositivos móviles para el aprendizaje en educación superior. Estudios sobre educación, 30, 175-195 (2016)

Lugo, M. T., y Schurmann, S. Activando el aprendizaje móvil en América Latina, UNESCO, 1(1), 9-46 (2012)

Mejía, J., y López, D. Modelo de Calidad de E-learning para Instituciones de Educación Superior en Colombia, doi:10.4067/S0718-50062016000200007, Formación Universitaria, 9(2), 59-72 (2016)

Montrieux, H., Vanderlinde, R., Schellens, T., y De Marez, L. Teaching and Learning with Mobile Technology: A Qualitative Explorative Study about the Introduction of Tablet Devices in Secondary Education, doi:10.1371/journal.pone.0144008, PLOS One, 10(12), 1-17 (2015)

Munch-Galindo, L., y Angeles, E. Métodos y técnicas de investigación, 1a edición, Trillas (1998)

Shuler, C., Winters, N., y West, M. El futuro del aprendizaje móvil, implicaciones para la planificación y la formulación de políticas, UNESCO, 1-49 (2013) 
Su, C. H., \& Cheng, C. H. A mobile gamification learning system for improving the learning motivation and achievements. Journal of Computer Assisted Learning, 31, 268-286 (2015)

UNESCO. Policy Guidelines for Mobile Learning (2013)

UNESCO. Las TIC en educación. (en la web: http://www.unesco.org/new/es/unesco/themes/icts/m4ed/, acceso: 16 de mayo 2016), (2013)

Vosloo, S. Aprendizaje móvil y políticas, UNESCO, 1(1), 3-59 (2013)

West, M., y Ei Chew, H. Reading in the mobile era. A study of mobile reading in developing countries, UNESCO (2015)

Yu-Chang, H., y Yu-Hui, C. A Review of Models and Frameworks for Designing Mobile Learning Experiences and Environments. CJLT, 41(3) (2015) 